BU/HEPP/96-02

June 1996

\title{
Lattice Charge Overlap II: Aspects of Charged Pion Polarizability
}

\author{
Walter Wilcox \\ Department of Physics, Baylor University, Waco, TX 76798-7316
}

\begin{abstract}
Formulas are developed for use in lattice studies of charged hadron polarizabilities. In particular, the valence quark different-flavor component of the charged pion polarizability is examined on a $16^{3} \times 24$ lattice at $\beta=6.0$ using Wilson fermions. Using the elastic limit results of Part I of this series, it is concluded that this represents a small negative component of the full charged polarizabilty.
\end{abstract}




\section{INTRODUCTION}

In the previous paper of this series, referred to here as Part I, the elastic limit of pions and rho mesons and were examined and the results were compared to lattice simulations using three-point function techniques. The elastic "baseline" set in Part I makes the measurement of nonelastic properties of these mesons possible.

Neutral hadron polarizabilities have been examined previously using the techniques of lattice QCD [1]. The authors there used an external field method to study the effect of a uniform electric field on the correlation functions of mesons and baryons. However, as of yet there have been no investigations of charged hadron polarizabilities using the lattice techniques. This is unfortunate since there is now a growing body of experimental information on this subject. Of course, external field methods would be very difficult or impossible to use in the case of charged hadrons because charged particles accelerate in an electric field. The definition of charged hadron polarizability takes the form of the response of the particle to an applied oscillating electromagnetic field. For this purpose a single photon will do and the effect being considered is then just Compton scattering.

The subject of charged pion polarizability will be considered from the point of view of an effective relativistic field theory. Comparing the general and phenomenological forms of the Compton scattering amplitude to second order in the photon momentum will give us the form of the polarizability coefficient. Next, the method for extracting polarizability on the lattice is explained. It is pointed out that the full pion polarizabilty requires the separate evaluation of five types of connected and disconnected fermion diagrams. In the fourth section the details of of our numerical simulation of the valence different-flavor component of the charged pion polarizabilty will be given and the results from Part I will be extensively used. It will be established that the resulting contribution is a small negative component of the full charged polarizabilty. 


\section{THEORY}

Charged hadron polarizability has been examined previously in Ref. [2] using the methods of charge overlap in the context of lattice QCD simulations. However, the methods of Ref. [2] are coordinate-space based; the present techniques [3] are momentum-space based. First, let us review the definition of charged pion polarizability as measured in Compton scattering. The definition of charged polarizability in terms of relativistic matrix elements in the context of an effective meson theory will then be established.

\section{A. Review}

The general form of the charged pion Compton scattering amplitude,

$$
T_{\mu \nu}=i \int d^{4} x e^{i k_{2} \cdot x}\left(\pi\left(p_{2}\right)\left|T\left(j_{\mu}(x) j_{\nu}(0)\right)\right| \pi\left(p_{1}\right)\right)
$$

where ' $T$ ' denotes time ordering and

$$
j_{\mu}(x)=q_{u} j_{\mu}^{u}(x)+q_{d} j_{\mu}^{d}(x)
$$

$\left(q_{u}=\frac{2}{3}, q_{d}=-\frac{1}{3}\right)$ is an electromagnetic current density for up and down quarks, to second order in the photon momentum is given by «, 4

$$
\begin{gathered}
\sqrt{2 E_{1} 2 E_{2}} T_{\mu \nu}=-\frac{T_{\mu}\left(p_{1}+k_{1}, p_{1}\right) T_{\nu}\left(p_{2}, p_{2}+k_{2}\right)}{\left(p_{1}+k_{1}\right)^{2}-m^{2}}-\frac{T_{\mu}\left(p_{2}, p_{2}-k_{1}\right) T_{\nu}\left(p_{1}-k_{2}, p_{1}\right)}{\left(p_{1}-k_{2}\right)^{2}-m^{2}} \\
+2 g_{\mu \nu}+A\left(k_{1}^{2} g_{\mu \nu}-k_{1 \mu} k_{1 \mu}+k_{2}^{2} g_{\mu \nu}-k_{2 \mu} k_{2 \mu}\right)+B\left(k_{1} \cdot k_{2} g_{\mu \nu}-k_{2 \mu} k_{1 \nu}\right)+C t_{\mu \nu} .
\end{gathered}
$$

In this context $A, B$ and $C$ are simply numerical coefficients. A noncovariant continuum state normalization,

$$
\left.\sum_{n} \int \frac{d^{3} p}{(2 \pi)^{3}} \mid n(p)\right)(n(p) \mid=\mathbf{1},
$$

is being used which is responsible for the square root factor involving pion energies appearing on the left hand side of Eq.(3). The pion electromagnetic vertex $\left(q_{\mu} \equiv p_{\mu}^{\prime}-p_{\mu}\right)$,

$$
T_{\mu}\left(p^{\prime}, p\right)=\left(p_{\mu}^{\prime}+p_{\mu}\right) F_{\pi}\left(q^{2}\right)+q_{\mu} \frac{p^{2}-p^{2}}{q^{2}}\left(1-F_{\pi}\left(q^{2}\right)\right),
$$


has been written so as to obey

$$
q_{\mu} T_{\mu}\left(p^{\prime}, p\right)=p^{2}-p^{2}
$$

for off-shell pions, which is needed in order to satisfy the scalar electromagnetic Ward identity [5]. To the fourth order in momentum (needed later),

$$
F_{\pi}\left(q^{2}\right)=1-\frac{<r^{2}>}{6} q^{2}+\frac{<r^{4}>}{120} q^{4} .
$$

In the above $m$ is the pion mass and the tensor $t_{\mu \nu}$ is given by

$$
t_{\mu \nu}=\left(k_{1} \cdot k_{2}\right) Q_{\mu} Q_{\nu}+\left(Q \cdot k_{1}\right)\left(Q \cdot k_{2}\right) g_{\mu \nu}-\left(Q \cdot k_{2}\right) Q_{\mu} k_{1 \nu}-\left(Q \cdot k_{1}\right) Q_{\nu} k_{2 \mu}
$$

$\left(Q_{\mu}=p_{1 \mu}+p_{2 \mu}.\right)$ The Compton amplitude is conserved $\left(k_{1}^{\mu} T_{\mu \nu}=k_{2}^{\nu} T_{\mu \nu}=0\right)$, which can be shown to result in $A=\frac{<r^{2}>}{3}$ to this order. The first two terms on the right of Eq.(3) represent elastic scattering from the pion while the others are contact terms. (A metric with $g^{00}=1, g^{i i}=-1,(i=1,2,3)$ is being used. $)$ In order to define electric and magnetic polarizabilities, let us examine the Compton scattering amplitude $\alpha \epsilon_{1}^{\mu} T_{\mu \nu} \epsilon_{2}^{\nu *}$, where $\alpha$ is the fine structure constant and $\epsilon_{1}^{\nu}$ and $\epsilon_{2}^{\nu *}$ are the initial and final photon polarization vectors, respectively. In the lab frame where the inital pion is at rest:

$$
\begin{aligned}
& k_{1} \cdot \epsilon_{1}=k_{2} \cdot \epsilon_{2}^{*}=0, \\
& p_{1} \cdot \epsilon_{1}=p_{1} \cdot \epsilon_{2}^{*}=0 .
\end{aligned}
$$

Choose $\epsilon_{1}$ and $\epsilon_{2}^{*}$ to have only spatial components. Defining $k_{(1,2)}^{0}=\omega_{(1,2)}$, in the nonrelativistic limit $\left(\left|\vec{k}_{(1,2)}\right|, \omega_{(1,2)}<<m\right)$, one can show for off-shell photons that

$$
\alpha \epsilon_{1}^{\mu} T_{\mu \nu} \epsilon_{2}^{\nu *}=\hat{\epsilon}_{1} \cdot \hat{\epsilon}_{2}^{*}\left[-\frac{\alpha}{m}\left(1+\frac{<r^{2}>}{6}\left(k_{1}^{2}+k_{2}^{2}\right)\right)+\alpha_{E} \omega_{1} \omega_{2}\right]+\beta_{M}\left(\hat{\epsilon}_{1} \times \vec{k}_{1}\right) \cdot\left(\hat{\epsilon}_{2}^{*} \times \vec{k}_{2}\right),
$$

where

$$
\begin{array}{r}
\alpha_{E} \equiv-\alpha\left(\frac{B}{2 m}+2 m C\right), \\
\beta_{M} \equiv \alpha \frac{B}{2 m},
\end{array}
$$

are by definition the electric and magnetic polarizabilities, respectively. An explicit form for $\alpha_{E}$ will now be exhibited and an explanation of how this coefficient can be measured in a lattice simulation will be given. 


\section{B. Derivation}

First of all, let us use the continuum to lattice corespondences,

$$
\begin{array}{r}
\mid n(p)) \longrightarrow V^{1 / 2} \mid n(p)> \\
j_{\mu}(x) \longrightarrow \frac{j_{\mu}^{L}(x)}{a^{3}} \\
\int d^{4} x \longrightarrow V a \int_{-\infty}^{\infty} d t \sum_{\vec{x}}
\end{array}
$$

where $V=N_{s} a^{3}, N_{s}$ being the number of spatial sites in the lattice and 'a' being the spacing, to rewrite Eq.(1) as

$$
T_{\mu \nu}=i N_{s}^{2} a \int d t \sum_{\vec{x}} e^{i k_{2} \cdot x}<p_{2}\left|T\left(j_{\mu}^{L}(x) j_{\nu}^{L}(0)\right)\right| p_{1}>
$$

Notice that time remains continuous (and is dimensionless) in this formalism and that a possible finite renormalization factor has been neglected in Eq.(15) since the exactly conserved lattice charge density will be used here.

Next, a point about the amplitude being calculated needs to be made. On the lattice, the appropriate object to consider is actually a normal ordered product of currents:

$$
T_{\mu \nu}^{e f f}=i N_{s}^{2} a \int d t \sum_{\vec{x}} e^{i k_{2} \cdot x}<\pi\left(p_{2}\right)\left|: j_{\mu}^{L}(x) j_{\nu}^{L}(0):\right| \pi\left(p_{1}\right)>
$$

This is because the vacuum amplitude lattice Green function used (see Eq.(36) below) contains a disconnected part when $p_{2}=p_{1}$ which must be removed by hand. The form of the disconnected amplitude being subtracted in Eq.(18) will later be exhibited explicitly.

The methods of charge overlap will be used to calculate the necessary amplitudes. Thus, we consider the four-point function corresponding to $T_{00}$ with the kinematical conditions:

$$
\omega_{1}=\omega_{2}=0 ; \quad p_{10}=p_{20}=m ; \quad\left|\vec{k}_{1}\right|=\left|\vec{k}_{2}\right|<<m
$$

The relation between normal-ordered and time-ordered currents is

$$
: j_{\mu}^{L}(x) j_{\nu}^{L}(0):=T\left(j_{\mu}^{L}(x) j_{\nu}^{L}(0)\right)-<0\left|T\left(j_{\mu}^{L}(x) j_{\nu}^{L}(0)\right)\right| 0>
$$

Using lattice completeness, 


$$
\sum_{n, \vec{p}_{n}}\left|n\left(\vec{p}_{n}\right)><n\left(\vec{p}_{n}\right)\right|=\mathbf{1}
$$

as well as space-time translations,

$$
j_{\mu}^{L}(x)=e^{i p \cdot x} j_{\mu}^{L}(0) e^{-i p \cdot x}
$$

the space sum and time integral in Eq.(18) now results in

$$
\left.T_{00}^{e f f}\right|_{\text {"kinematics" }}=2 N_{s}^{2} \sum_{n} \frac{|<\pi(\overrightarrow{0})| \rho^{L}(0)|n(\vec{k})>|^{2}}{\left(E_{n}-m\right)}-2 \frac{N_{s}}{a^{3}} \sum_{n} \frac{|<0| \rho^{L}(0)|n(\vec{k})>|^{2}}{E_{n}} .
$$

Contact between Eq.(23) and Eq.(3) above now needs to be made. Separate off the elastic part in Eq.(23),

$$
\begin{gathered}
T_{00}^{e l} \equiv 2 N_{s}^{2} \frac{|<\pi(\overrightarrow{0})| \rho^{L}(0)|\pi(\vec{k})>|^{2}}{\left(E_{\pi}-m\right)}, \\
<\pi(\overrightarrow{0})\left|\rho^{L}(0)\right| \pi(\vec{k})>=\frac{1}{N_{s}} \frac{\left(E_{\pi}+m\right)}{\sqrt{2 E_{\pi} 2 m}} F_{\pi}\left(q^{2}\right),
\end{gathered}
$$

where $F_{\pi}\left(q^{2}\right)$ is the usual continuum pion form factor. Using Eq.(7), one can show after some algebra that

$$
T_{00}^{e l}=\frac{4 m}{\vec{k}^{2}}+\left\{\frac{1}{m}-\frac{4}{3} m<r^{2}>\right\}+\vec{k}^{2}\left\{-\frac{<r^{2}>}{3 m}+\frac{1}{9} m<r^{2}>^{2}+\frac{1}{15} m<r^{4}>\right\},
$$

up to second order in the photon momentum. For the nonelastic part one may show at low momentum $(|\vec{k}|<<m)$ for vector or axial vector states that

$$
\begin{aligned}
& <\pi(\overrightarrow{0})\left|\rho^{L}(0)\right| n(\vec{k})>=\frac{\epsilon^{0}(k) d_{\pi n} m_{n}}{N_{s}}, \\
& <0\left|\rho^{L}(0)\right| n(\vec{k})>=\frac{\epsilon^{0}(k) d_{0 n} m_{n} a^{1 / 2}}{\sqrt{N_{s}}}
\end{aligned}
$$

which serves to define the constants $d_{\pi n}$ and $d_{0 n}$, and $\epsilon^{0}(k)$ is the 0 th component of a polarization vector for the state $\mid n(\vec{k})>$ with mass $m_{n}$. The $d_{\pi n}, d_{\pi 0}$ factors are defined in these expressions so that they are independent of the lattice size and spacing. Interestingly, vectors and axial vectors are the only types of intermediate states which may contribute to the polarizability. See the Appendix for further details. If we choose a helicity basis where 
the $\epsilon^{\mu}(k, \lambda)$ with $\lambda=+,-$ are purely spatial and the positive-z axis is along $\vec{k}$, then only the longitudinal polarization state, $\lambda=0$, contributes in Eqs.(27) and (28),

$$
\epsilon^{0}(k, 0)=\frac{|\vec{k}|}{m_{n}}
$$

and the expansion to second order in photon momentum for these kinematics then yields

$$
T_{00}^{e f f} \mid \text { "kinematics" }=T_{00}^{e l}+2 \vec{k}^{2}\left(\sum_{n \neq \pi} \frac{\left|d_{\pi n}\right|^{2}}{\left(E_{n}-m\right)}-V \sum_{n} \frac{\left|d_{0 n}\right|^{2}}{E_{n}}\right),
$$

where $T_{00}^{e l}$ is given by Eq.(26).

The phenomenological expression, Eq.(30), is to be compared with the $\mu=\nu=0$ component of the general Compton amplitude, Eq.(3), also to order $\vec{k}^{2}$ with the same kinematics. One may show that

$$
\begin{aligned}
2 m T_{00} \mid \text { "kinematics" }= & \frac{T_{0}\left(p_{1}+k_{1}, p_{1}\right) T_{0}\left(p_{2}, p_{2}+k_{2}\right)}{\left(p_{1}+k_{1}\right)^{2}-m^{2}}-\frac{T_{0}\left(p_{2}, p_{2}-k_{1}\right) T_{0}\left(p_{1}-k_{2}, p_{1}\right)}{\left(p_{1}-k_{2}\right)^{2}-m^{2}} \\
& +2-\frac{2<r^{2}>\vec{k}^{2}-B \vec{k}^{2}-4 m^{2} \vec{k}^{2} C .}{3}
\end{aligned}
$$

For these kinematics, one has

$$
\frac{1}{\left(p_{1}+k_{1}\right)^{2}-m^{2}}=-\frac{1}{\left(p_{1}-k_{2}\right)^{2}-m^{2}}=-\frac{1}{\vec{k}^{2}},
$$

and also, for example,

$$
T_{0}\left(p_{1}+k_{1}, p_{1}\right)=2 m\left(1-\frac{<r^{2}>}{6} q^{2}+\frac{<r^{4}>}{120} q^{4}\right) .
$$

When the pieces are put together, one obtains

$$
\begin{aligned}
T_{00} \mid \text { "kinematics" }= & \frac{4 m}{\vec{k}^{2}}+\left\{\frac{1}{m}-\frac{4}{3} m<r^{2}>\right\}+\vec{k}^{2}\left\{-\frac{<r^{2}>}{3 m}+\frac{1}{9} m<r^{2}>^{2}\right. \\
& \left.+\frac{1}{15} m<r^{4}>-\left(\frac{B}{2 m}+2 m C\right)\right\} .
\end{aligned}
$$

As the last step in the derivation, compare Eq.(30) and Eq.(34) and identify

$$
\alpha_{E}=-\alpha\left(\frac{B}{2 m}+2 m C\right)=2 \alpha\left\{\sum_{n \neq \pi} \frac{\left|d_{\pi n}\right|^{2}}{\left(E_{n}-m\right)}-V \sum_{n} \frac{\left|d_{0 n}\right|^{2}}{E_{n}}\right\} .
$$


This formula relates $\alpha_{E}$ to properties of relativistic matrix elements, which was the goal. Of course, the $d_{\pi n}$ and $d_{0 n}$ factors are nothing but appropriate derivatives of the matrix elements in Eq. (28) with respect to $|\vec{k}|$, evaluated at $\vec{k}=0$. There are several other aspects of this formula worthy of mention. First, the explicit appearance of a volume factor makes it clear that neither term above is finite, but the whole expression must be regulated (as on the lattice) to extract a finite answer. Second, it does not have the classical charge radius term, $\alpha<r^{2}>/ 3 m$. This is because it comes from comparing to an effective theory of point mesons. (Even the pion is viewed as a point object in this case; it's "size" simply comes from additional local interactions with photons.) Third, it explicitly includes the vacuum polarization contribution first explained by Terent'ev in Ref. [6]. The negative sign in front of this term makes it abundantly clear why in relativistic field theories the charged particle's polarizability can be positive or negative. The polarizability of the pion, no longer considered the groundstate as in nonrelativistic quantum mechanics, is actually defined relative to the vacuum, a result remininiscent of the Casimir effect. Finally, even though we are only keeping terms to order $\vec{k}^{2}$, it is clear that the above is an exact formula for the polarizability, which is simply the numerical coefficient of the second order energy term in Eq.(11).

\section{LATTICE MEASUREMENT}

Let us concentrate now on the lattice amplitudes necessary to directly extract the charged pion polarizability. Some comments about indirect lattice measurements of this quantity will be made later.

For these purposes, one forms the usual charge overlap function, but with normal ordering of the charge densities instead of time ordering:

$$
P_{\pi}\left(\vec{r} ; t_{3}, t_{2}, t_{1}\right) \equiv \frac{\sum_{\vec{x}_{3}, \vec{z}}<0\left|\phi^{\dagger}\left(x_{3}\right): \rho^{L}\left(x_{2}\right) \rho^{L}\left(x_{1}\right): \phi(z)\right| 0>}{\sum_{\vec{x}_{3}, \vec{z}}<0\left|\phi^{\dagger}\left(x_{3}\right) \phi(z)\right| 0>}
$$

where $t_{3}>\left(t_{2}, t_{1}\right)>0, z=(0, \vec{z})$ and $r \equiv x_{2}-x_{1}$. Dimensionless times and distances 
are being used in this section. The formulas are now in discrete Euclidean space but one imagines the time axis is still continuous. The time limits $t_{3}>>t_{1,2}>>1$ yield

$$
P_{\pi}\left(\vec{r} ; t_{3}, t_{2}, t_{1}\right) \longrightarrow<\pi(\overrightarrow{0})\left|: \rho^{L}\left(x_{2}\right) \rho^{L}\left(x_{1}\right):\right| \pi(\overrightarrow{0})>
$$

where

$$
\begin{aligned}
<\pi(\overrightarrow{0})\left|: \rho^{L}\left(x_{2}\right) \rho^{L}\left(x_{1}\right):\right| \pi(\overrightarrow{0})>= & \left.<\pi(\overrightarrow{0}) \mid T\left(\rho^{L}(r) \rho^{L} 0\right)\right) \mid \pi(\overrightarrow{0})> \\
& -<0\left|T\left(\rho^{L}(r) \rho^{L}(0)\right)\right| 0>.
\end{aligned}
$$

Consider now the finite Fourier transform of $P_{\pi}\left(\vec{r} ; t_{3}, t_{2}, t_{1}\right)$,

$$
Q\left(\vec{q} ; t_{3}, t_{2}, t_{1}\right) \equiv N_{s} \sum_{\vec{r}} e^{-i \vec{q} \cdot \vec{r}} P_{\pi}\left(\vec{r} ; t_{3}, t_{2}, t_{1}\right)
$$

In the same time limit:

$\left.Q\left(\vec{q} ; t_{3}, t_{2}, t_{1}\right) \longrightarrow N_{s} \sum_{\vec{r}} e^{-i \vec{q} \cdot \vec{r}}\left\{<\pi(\overrightarrow{0}) \mid T\left(\rho^{L}(r) \rho^{L} 0\right)\right)|\pi(\overrightarrow{0})>-<0| T\left(\rho^{L}(r) \rho^{L}(0)\right) \mid 0>\right\}$

Assuming $t_{2}-t_{1}>0$ and inserting a complete set of states now gives:

$$
\begin{aligned}
Q\left(\vec{q} ; t_{3}, t_{2}, t_{1}\right) \longrightarrow & N_{s}^{2}\left\{\sum_{n}|<\pi(\overrightarrow{0})| \rho^{L}(0)|n(\vec{q})>|^{2} e^{-\left(E_{n} a-m_{\pi} a\right)\left(t_{2}-t_{1}\right)}\right. \\
& \left.-\sum_{n}|<0| \rho^{L}(0)|n(\vec{q})>|^{2} e^{-E_{n} a\left(t_{2}-t_{1}\right)}\right\} .
\end{aligned}
$$

Separate off the elastic term $(n=\pi)$,

$$
Q^{e l}\left(\vec{q} ; t_{2}-t_{1}\right) \equiv N_{s}^{2}|<\pi(\overrightarrow{0})| \rho^{L}(0)|\pi(\vec{q})>|^{2} e^{-\left(E_{\pi} a-m_{\pi} a\right)\left(t_{2}-t_{1}\right)}
$$

above. Then, using Eqs.(27), (28) and (29) at low momentum transfer results in the construction,

$$
\begin{aligned}
& \alpha a \int_{-\infty}^{\infty} d\left(t_{2}-t_{1}\right)\left[Q\left(\vec{q} ; t_{3}, t_{2}, t_{1}\right)-Q^{e l}\left(\vec{q} ; t_{2}-t_{1}\right)\right] \\
& =2 \alpha \vec{k}^{2}\left(\sum_{n \neq \pi} \frac{\left|d_{\pi n}\right|^{2}}{\left(E_{n}-m\right)}-V \sum_{n} \frac{\left|d_{0 n}\right|^{2}}{E_{n}}\right)=\vec{k}^{2} \alpha_{E}
\end{aligned}
$$

$(\vec{k}=\vec{q} / a)$ when the relative time integral between the two charge densities is explicitly done. (One gets a doubling of the result when the other time relationship, $t_{2}-t_{1}<0$, is 
assumed.) Of course on a finite lattice the time integrals do not really extend to $\pm \infty$ because of the fixed time locations of the particle sources, but instead to time limits such that the elastic limit is effectively established. Eq.(43) now gives us the means of measuring the Compton scattering pion polarizability coefficient. On the lattice one may take a numerical "derivative" of the left hand side of Eq.43) with respect to $\vec{q}^{2}$ by simply dividing it by $\vec{q}^{2}$ at the lowest lattice momentum. (The relative error in this procedure is of order $\vec{q}^{2}$.)

It is crucial to work in momentum space to extract the polarizability. In position space one would look at (image corrections are assumed),

$$
R^{2}\left(t_{3}, t_{2}, t_{1}\right) \equiv \sum_{\vec{r}} \vec{r}^{2} P_{\pi}\left(\vec{r} ; t_{3}, t_{2}, t_{1}\right)
$$

which does not project onto good momentum when a complete set of states is inserted [2]. Thus, the limitation to small momenta, necessary to use Eqs.(27) and (28) above, can not be enforced.

Although Eq.(43) is a simple formula, it's direct evaluation on the lattice via four-point functions which arise from the field contractions in Eq.(36) is not easy. There are five types of diagrams, which may be classified by fermion line topology, as shown in Fig.1. In the case of Figs.1(a) and (b), the photons interact directly with the valence quarks. Fig.1(a) will be termed the same-flavor valence part and Fig.1(b) will be called the different-flavor valence part. Figs.1(c), (d) and (e) represent contributions from disconnected fermion lines in which arbitrary numbers of gluon lines connect the various pieces together. These gluon connections are enforced in the correlations between the pion propagator and the various fermion loops in the lattice Monte Carlo. (When isolated groups of fermion lines arise, "connected part" means "statistically correlated part" on the lattice.) Fig.1(c) represents the contribution from correlations between a valence quark and a self-contracted quark loop. (Flavors other than $u$ and $d$ can of course contribute to the quark loops.) Fig.1(d) has both currents, either same-flavor or different-flavor, self-contracted. In the case of Fig.1(e), one has a complete quark loop formed. It is the amplitude where the pion propagator is completely disconnected from the quark loops in (d) and (e) (when $p_{2}=p_{1}$ ) which 
needs explicit subtraction and is responsible for the negative contribution on the right hand side of Eq.(35). Fig.1(c) also has a disconnected part, however, there is no need to do a subtraction since it can be shown that the vacuum expectation value of the exactly conserved lattice charge density vanishes configuration by configuration for color $\mathrm{SU}(2)$, and in the configuration average for $\mathrm{SU}(3)$.

The lattice formula for $\alpha_{E}$ has now been derived and the agenda for evaluating the charged pion polarizability directly on the lattice has been set. In the next section we will examine one particular type of diagram and show that it actually makes only a small contribution to $\alpha_{E}$.

\section{LATTICE SIMULATION}

As pointed out immediately above, the direct calculation of the charged pion polarizability involves many diagrams which must be separately evaluated. In this section the different-flavor valence piece, Fig.1(a), will be considered.

The full different-flavor piece involves Figs.1(a), (c) and (d) and can be evaluated on the lattice at low lattice momentum, $\vec{q}$, by

$$
\alpha_{E}^{u, d}=\frac{\alpha a^{3}}{\vec{q}^{2}} \int_{-\infty}^{\infty} d\left(t_{2}-t_{1}\right)\left[Q^{u, d}\left(\vec{q} ; t_{3}, t_{2}, t_{1}\right)-Q^{e l ; u, d}\left(\vec{q} ; t_{2}-t_{1}\right)\right]
$$

where $Q^{u, d}\left(\vec{q} ; t_{3}, t_{2}, t_{1}\right)$ and $Q^{e l ; u, d}\left(\vec{q} ; t_{2}-t_{1}\right)$ are identified from the Fourier transform of

$$
P_{\pi}^{u, d}\left(\vec{r} ; t_{3}, t_{2}, t_{1}\right) \equiv 2 q_{u} q_{d} \frac{\sum_{\vec{x}_{3}, \vec{z}}<0\left|\phi^{\dagger}\left(x_{3}\right): \rho^{L ; u}\left(x_{2}\right) \rho^{L ; d}\left(x_{1}\right): \phi(z)\right| 0>}{\sum_{\vec{x}_{3}, \vec{z}}<0\left|\phi^{\dagger}\left(x_{3}\right) \phi(z)\right| 0>},
$$

as above.

We now specialize to evaluating the valence $u, d$ contribution from Fig.1(a). We refer the reader to Part I for a discussion of the details of the numerical simulation, which was carried out on 20 quenched $16^{3} \times 24$ lattices with Wilson fermions at $\beta=6.0$. The appropriate correlation functions for three quark masses are displayed in Fig.5 of Part I. According to Eq.(43), we now need to evalute the area under these functions and subtract the elastic 
contribution. The results of this procedure are presented in Table I, where the errors specified are purely statistical. In arriving at these results, the numerical value $\alpha a^{3}=1.06 \times 10^{-44} \mathrm{~cm}^{3}$ has been used, where $\alpha$ is the fine structure constant. This value is inferred from the inverse lattice spacing, $a^{-1}=1740 \mathrm{MeV}$, which is taken from comparing the chirally extrapolated lattice nucleon mass with the experimental value [8].

When the results in Table I are extrapolated linearly in quark mass, as defined in Part I, to the chiral limit at $\kappa_{c r}=0.1564$, we obtain $\left(\alpha_{E}^{u, d}\right)^{\text {valence }}=-.12(11) \times 10^{-4} \mathrm{fm}^{3}$.

There are various systematic uncertainties in our final numerical result. One systematic uncertainty is associated with the numerical evaluation of the discrete nonelastic time correlation integral. This discrete time integral was done using both the trapezoidal rule and Simpson's method [0]. The differences for all three $\kappa$ values were less than $2 \%$. There is an additional systematic uncertainty associated with the numerical momentum derivative. As pointed out above, this is expected to be of order $\vec{q}^{2}$, or about $10 \%$, the same as other finite lattice spacing errors. This was examined numerically by repeating the simulation (with the same fits) on 10 configurations, also at $\beta=6.0$, on a larger $20^{3} \times 30$ lattice at $\kappa=.152$. The

result on the larger lattices is $-.33(14) \times 10^{-4} \mathrm{fm}^{3}$, which unfortunately has large statistical errors, but is consistent with the value in Table I. In addition to these uncertainties, there is also the systematic uncertainty associated with the setting of the lattice scale. Since the pion polarizability scales as $a^{3}$, it is rather sensitive to a change in the scale. In order to remain consistent with the low energy lattice results for nucleon mass, pion decay constant and proton electric form factor in Ref. [8], a roughly $10 \%$ change in the present lattice scale could be tolerated. This means a systematic scale uncertainty of approximately $33 \%$ for $\alpha_{E}$.

\section{SUMMARY AND CONCLUSIONS}

The establishment of the charge overlap elastic limit in Part I allows the possibility of obtaining nonelastic hadron properties directly from lattice QCD. In particular, using the lattice formulas developed, we have see that the charged pion polarizability can be measured 
on the lattice from low momentum charge overlap correlation functions.

We have obtained $\left(\alpha_{E}^{u, d}\right)^{\text {valence }}=-.12(11) \times 10^{-4} \mathrm{fm}^{3}$ in the chiral limit from the quark diagram in Fig.1(a). This is to be compared with the experimental result 9]

$$
\alpha_{E}^{e x p}=2.2(16) \times 10^{-4} \mathrm{fm}^{3},
$$

and the result from second order chiral pertubation theory [10],

$$
\alpha_{E}^{\text {chiral }}=2.4(5) \times 10^{-4} \mathrm{fm}^{3} .
$$

Thus, the present results establish that the valence quark different-flavor diagram, Fig.1(a) contributes only a small, negative component to the full charged pion polarizability. We must therefore look to the other diagrams in Fig.1 for the bulk of this effect.

It is important to note that in addition to the present, direct measurement of the charged pion polarizability, there is also the Das, Mathur, Okubo (DMO) current algebra sum rule 111 for $\alpha_{E}$. Although this formula is not exact, the measurements required for it's evaluation on the lattice are much simpler than the direct method since it involves only mesonic twopoint functions. A lattice evaluation of the so-called intrinsic part of the charged pion polarizability in the DMO sum rule should be quite interesting and is currently underway.

Finally, it was noted above that the lattice measurement of $\alpha_{E}$ scales like $a^{3}$ and is therefore quite sensitive to the lattice scale. This opens up the possibility of using charged pion polarizability as a place to accurately test QCD, or alternately, to set the lattice scale, once the lattice and experimental results are both improved.

\section{ACKNOWLEDGMENTS}

This work is supported in part by the NSF under Grant No. PHY-9401068. It is also supported by the National Center for Supercomputing Applications (NCSA) at the University of Illinois at Urbana-Champaign and used the CM5 and SGI Power Challenge computers. 


\section{Appendix}

In this Appendix it is established that the only intermediate states which may contribute in Eq.(35) to the charged pion polarizability are vectors and axial vectors.

First, let us dispose of the spin zero case. We have $(n \neq \pi)$

$$
\left(\pi(p)\left|j_{\alpha}(0)\right| n\left(p_{n}\right)\right)=\frac{p_{\alpha} G_{1}\left(q^{2}\right)+p_{n \alpha} G_{2}\left(q^{2}\right)}{\left(2 E_{\pi} 2 E_{n}\right)^{1 / 2}} .
$$

where as usual $q^{2}=\left(p-p_{n}\right)^{2}$. Charge conservation requires the right hand side of Eq.(47) dotted into $p_{n}^{\mu}-p^{\mu}$ to be zero, so that

$$
G_{2}\left(q^{2}\right)=G_{1}\left(q^{2}\right)\left(\frac{m^{2}-p \cdot p_{n}}{m_{n}^{2}-p \cdot p_{n}}\right) .
$$

Therefore (considering the pion at zero spatial momentum)

$$
\left(\pi(\overrightarrow{0})\left|j_{4}(0)\right| n\left(p_{n}\right)\right)=\frac{G_{1}\left(q^{2}\right)}{\left(2 m 2 E_{n}\right)^{1 / 2}}\left(m+E_{n} \frac{m^{2}-m E_{n}}{m_{n}^{2}-m E_{n}}\right) .
$$

At low momentum, $\left|\vec{p}_{n}\right|<<m$, we then find

$$
\left(\pi(\overrightarrow{0})\left|j_{0}(0)\right| n\left(p_{n}\right)\right) \propto G_{1}\left(\left(m-m_{n}\right)^{2}\right) \vec{p}_{n}^{2} .
$$

Therefore the absolute square of this matrix element goes at least like $\vec{p}_{n}^{4}$ at low momentum,

assuming $G_{1}\left(\left(m-m_{n}\right)^{2}\right)$ is nonsingular, and can not contribute to the pion polarizability term in $T_{00}^{e f f}$, which is proportional to $\vec{p}_{n}^{2}$.

We now consider general nonzero spin, s. Some fundamental properties of polarization tensors are: $\left(\lambda_{s}=-s,,, 0,, s\right)$ :

$$
\begin{aligned}
\epsilon^{\mu_{1} \cdots \mu_{s}}\left(p, \lambda_{s}\right) \epsilon_{\mu_{1} \cdots \mu_{s}}^{*}\left(p, \lambda_{s}^{\prime}\right) & =\delta_{\lambda_{s}, \lambda_{s}^{\prime}}, \\
g_{\mu_{\alpha} \mu_{\beta}} \epsilon^{\mu_{1} \cdots \mu_{\alpha} \cdots \mu_{\beta} \cdots \mu_{s}}\left(p, \lambda_{s}\right) & =0, \\
p_{\mu_{\alpha}} \epsilon^{\mu_{1} \cdots \mu_{\alpha} \cdots \mu_{s}}\left(p, \lambda_{s}\right) & =0 .
\end{aligned}
$$

Adopting a helicity basis, the Lorentz structure for spin s must be of the form

$$
\left(\pi(p)\left|j_{\alpha}(0)\right| n\left(s, \lambda_{s}, p_{n}\right)\right)=\frac{\epsilon^{\mu_{1} \cdots \mu_{s}}\left(p_{n}, \lambda_{s}\right) A_{\mu_{1} \cdots \mu_{s} \alpha}\left(p, p_{n}\right)}{\left(2 E_{\pi} 2 E_{n}\right)^{1 / 2}} .
$$


where it is assumed that the tensor $A_{\mu_{1} \cdots \mu_{s} \alpha}\left(p, p_{n}\right)$ is nonsingular.

First, we will need the following result:

$$
\left.\begin{array}{c}
\epsilon^{0 \cdots 0}\left(p, \lambda_{s}\right) \propto|\vec{p}|^{s}, \quad \lambda_{s}=0, \\
\epsilon^{0 \cdots 0}\left(p, \lambda_{s}\right)=0, \quad \lambda_{s} \neq 0 .
\end{array}\right\}
$$

This can be established from the higher spin considerations in Ref. [12]. This reference shows that the polarization vectors for higher spin may be constructed from those of spin 1 via

$$
\sum_{\lambda_{s}=-s}^{s} \psi_{s \lambda_{s}}(\xi) \epsilon^{\mu_{1} \cdots \mu_{s}}\left(p, \lambda_{s}\right) x_{\mu_{1}} \cdots x_{\mu_{s}}=\left[\sum_{\lambda_{1}=-1}^{1} \psi_{1 \lambda_{1}}(\xi) \epsilon^{\mu}\left(p, \lambda_{1}\right) x_{\mu}\right]^{s}
$$

where

$$
\psi_{s \lambda_{s}}(\xi) \equiv\left[\frac{(2 s) !}{\left(s+\lambda_{s}\right) !\left(s-\lambda_{s}\right) !}\right]^{1 / 2} \xi_{+}^{s+\lambda_{s}} \xi_{-}^{s-\lambda_{s}}
$$

Using a purely timelike $x_{\mu}$ and recalling that $\epsilon^{0}\left(p, \lambda_{1}\right) \neq 0$ only when $\lambda_{1}=0$, it is easy to see by matching coefficients of $\xi_{+}$and $\xi_{-}$on both sides of Eq.56 that

$$
\left.\begin{array}{c}
\epsilon^{0 \cdots 0}\left(p, \lambda_{s}\right) \propto\left(\epsilon^{0}(p, 0)\right)^{s}, \quad \lambda_{s}=0 \\
\epsilon^{0 \cdots 0}\left(p, \lambda_{s}\right)=0, \quad \lambda_{s} \neq 0
\end{array}\right\}
$$

Now using $\epsilon^{0}(p, 0) \propto|\vec{p}|$ from Eq.(29) establishes Eq.(55) above. There are many other ways of proving this result.

Working in the rest frame of the pion and considering only $j_{0}$, the matrix element in Eq.(54) is now seen to be proportional to $\epsilon^{0 \cdots 0}\left(p, \lambda_{s}\right)$ :

$$
\left(\pi(\overrightarrow{0})\left|j_{0}(0)\right| n\left(s, \lambda_{s}, p_{n}\right)\right) \propto \epsilon^{0 \cdots 0}\left(p_{n}, \lambda_{s}\right)
$$

This comes about through repeated use of Eqs.(52) and (53). For example, consider the $s=2$ case. Explicitly, one has

$$
\begin{aligned}
\left(\pi(\overrightarrow{0})\left|j_{0}(0)\right| n\left(s, \lambda_{s}, p_{n}\right)\right)= & \frac{1}{\left(2 m 2 E_{n}\right)^{1 / 2}}\left(\epsilon^{i_{1} i_{2}} A_{i_{1} i_{2} 0}+\epsilon^{i_{1} 0} A_{i_{1} 00}\right. \\
& \left.+\epsilon^{0 i_{2}} A_{0 i_{2} 0}+\epsilon^{00} A_{000}\right) .
\end{aligned}
$$


The remaining spatial $O(3)$ symmetry of the matrix element requires that $A_{i_{1} 00} \propto\left(p_{n}\right)_{i_{1}}$, $A_{0 i_{2} 0} \propto\left(p_{n}\right)_{i_{2}}$, and $A_{i_{1} i_{2} 0} \propto\left(p_{n}\right)_{i_{1}}\left(p_{n}\right)_{i_{2}}$ or $A_{i_{1} i_{2} 0} \propto g_{i_{1} i_{2}}$. Through the use of Eqs.(52) and (53) each term in Eq. 600) is seen to be proportional to $\epsilon^{00}\left(p_{n}, \lambda_{2}\right)$. This in turn means that the matrix element couples only to $\lambda_{2}=0$ and behaves like $\vec{p}_{n}^{2}$ (or possibly higher power) at low momentum from Eq.(55). This argument can be extended to higher spin. Thus, $s=1, \lambda_{1}=0$ (axial) is the only possible contributor to the charged pion polarizability through this matrix element.

Although only the matrix element $\left(\pi(\overrightarrow{0})\left|j_{0}(0)\right| n\left(s, \lambda_{s}, p_{n}\right)\right)$ has been discussed, similar conclusions hold for $\left(0\left|j_{0}(0)\right| n\left(s, \lambda_{s}, p_{n}\right)\right)$; namely, that the state $n\left(s, \lambda_{s}, p_{n}\right)$ contributes to charged pion polarizability only when $s=1, \lambda_{1}=0$ (vector). 


\section{REFERENCES}

[1] H. R. Fiebig, W. Wilcox, and R. M. Woloshyn, Nucl. Phys. B324 (1989) 47.

[2] M. Burkardt, J. M. Grandy and J. W. Negele, Ann. Phys. 238 (1995) 441.

[3] W. Wilcox, Phys. Lett. B289 (1992) 411.

[4] J. F. Donoghue and B. F. Holstein, Phys. Rev. D40 (1989) 2378.

[5] M. V. Terent'ev, Sov. J. of Nucl. Phys. 16 (1973) 87.

[6] M. V. Terent'ev, Sov. J. of Nucl. Phys. 16 (1973) 576.

[7] P. J. Davis and I. Polonsky, in "Handbook of Mathematical Functions" (M. Abramowitz and I. Stegun, Eds.) Ch. 25, U. S. Dept. of Commerce, 1972.

[8] K. F. Liu, S. J. Dong, T. Draper and W. Wilcox, Phys. Rev. Lett. 74 (1995) 2172.

[9] D. Babusci et. al. Phys. Lett. B277 (1992) 158 .

[10] U. Bürgi, "Charged Pion-Pair Production and Pion Polarizabilities to Two Loops, hepph/9602429 (unpublished).

[11] T. Das, V. S. Mathur and S. Okubo, Phys. Rev. Lett. 19, (1967) 859.

[12] J. Schwinger, "Particle, Sources, and Fields", Vol. 1, Section 2-5, Addison-Wesley, 1970. 


\section{Table}

TABLE I. Valence contribution to the dfferent-flavor charged pion polarizability as a function of $\kappa$.

\begin{tabular}{cc}
\hline \hline$\kappa$ & $\alpha_{E}\left(\mathrm{fm}^{3}\right)$ \\
\hline 0.148 & $-0.29(6) \times 10^{-4}$ \\
0.152 & $-0.23(6) \times 10^{-4}$ \\
0.154 & $-0.15(10) \times 10^{-4}$ \\
\hline \hline
\end{tabular}




\section{Figure Caption}

1. Graphical representation of the four-point functions contributing to charged pion polarization. The zero-momentum pion interpolating fields are represented by horizontal bars, the time axis runs horizontally and the vertical direction represents one space dimension. (a) and (b) represent different-flavor and same-flavor valence contributions, respectively. Fig.1(c) is the contribution from a single isolated quark loop, while (d) is the two quark loop contribution. (e) arises from a same-flavor current-current contraction. It is assumed that gluon lines connect the various fermion lines together in each

diagram. Diagrams (d) and (e) have a disconnected part which must be subtracted. 


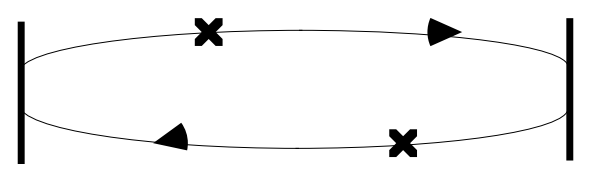

(a)

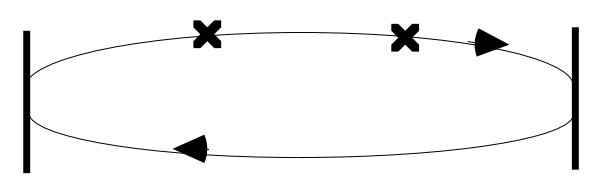

(b)

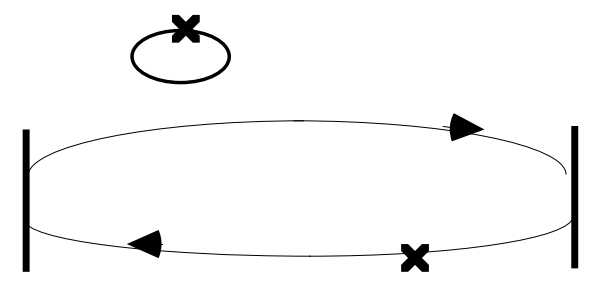

(c)

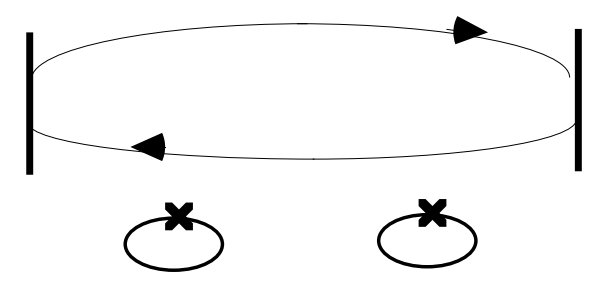

(d)

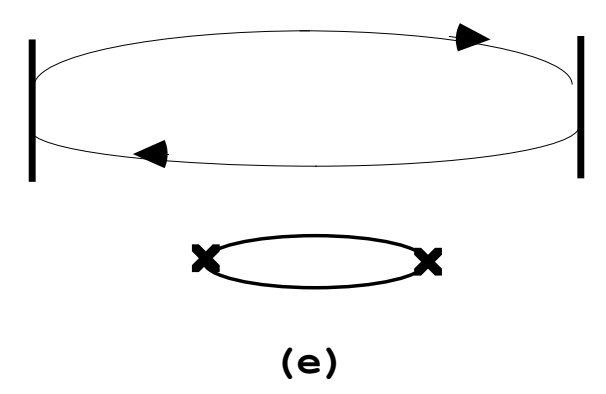

Fig.1 